\title{
Heart Rate Variability-Measured Stress and Academic Achievement in Medical Students
}

\author{
Hyo Hyun Yoo ${ }^{a}$ So Jung Yune ${ }^{b}$ Sun Ju Im ${ }^{b}$ Bee Sung Kam ${ }^{b}$ Sang Yeoup Lee ${ }^{b}$ c \\ aDepartment of Medical Education, Chonbuk National University School of Medicine, Jeonju, Republic of Korea; \\ ${ }^{b}$ Department of Medical Education, Pusan National University School of Medicine, Yangsan, South Korea; ' ${ }^{\top}$ Family \\ Medicine Clinic and Research Institute of Convergence of Biomedical Science and Technology, Pusan National \\ University Yangsan Hospital, Yangsan, South Korea
}

\section{Highlights of the Study}

- We studied the relationship between stress and academic achievement in medical students.

- Heart rate variability (HRV) is impacted by stress and is used for the objective assessment of stress.

- Medical students with higher stress measured by HRV have higher academic achievement, especially in written examinations.

\section{Keywords}

Clinical sciences · General practice · Primary health care · Medical education

\begin{abstract}
Objectives: Stress can affect learning and memory in students. Previous studies on stress in students were conducted mainly through surveys. We investigated how heart rate variability (HRV)-measured stress is related to academic achievement in medical students during clerkship. Subjects and Methods: A cross-sectional study was conducted. HRV measurements were performed in 97 third-year medical students during their family medicine clerkship course. Data on written and skilled examination scores of the end of the school year were also collected. We assessed the association between HRV-measured stress and written/skilled examina-
\end{abstract}

karger@karger.com www.karger.com/mpp

Karger $\stackrel{\text { ' }}{5}$

GOPEN ACCESS
(C) 2020 The Author(s)

Published by S. Karger AG, Basel

This is an Open Access article licensed under the Creative Commons Attribution-NonCommercial-4.0 International License (CC BY-NC) (http://www.karger.com/Services/OpenAccessLicense), applicable to the online version of the article only. Usage and distribution for commercial purposes requires written permission. tion scores. Results: Written examination scores showed a positive correlation with standard deviation of the normalto-normal intervals (SDNNs) $(r=0.245, p=-0.016)$, sympathetic nervous system/parasympathetic nervous system (SNS/PNS) balance ( $r=0.218, p=0.033)$, and stress index ( $r=$ $0.381, p=0.004)$. Students with an unhealthy SDNN, a dominant SNS, and a high stress index had higher scores in written examinations than students with a healthy SDNN, a balanced SNS/PNS, and a normal stress index, respectively $(p=$ $0.004,0.018$, and 0.012 , respectively). Moreover, skilled examination scores were negatively correlated with BMI $(r=$ $-0.249, p=0.014)$ and were higher in female students ( $r=$ $0.240, p=0.018$ ). Students with an abnormal autonomic balance diagram $(A B D)$ had a higher score than students with a normal $\operatorname{ABD}(p=0.03)$. Conclusions: This study shows that medical students with higher stress measured by HRV have higher academic achievement, especially in written exami- 
nations. Further studies are needed to confirm the results of this study and to assess the long-term effects of HRV-measured stress on medical students. 02020 The Author(s)

Published by S. Karger AG, Basel

\section{Introduction}

Globally, medical students experience a high degree of stress related to their academic load, such as a tremendous amount of learning, long semesters, a large number of examinations, and anxiety about a pass/fail grading system [1]. They also experience problems such as insufficient rest, low self-esteem, lack of sleep, and depressive mood. As a result, they suffer from a higher level of stress than college students of the same age in other disciplines [2]. However, the level of perceived stress varies depending on how they control and evaluate stressful situations or factors. If students perceive stress within the acceptable range, it can be helpful and motivating to do academic work as a coping mechanism $[3,4]$.

Stress is largely assessed by using questionnaires and by measuring objective physical responses [5-10]. Another method is to evaluate physiological and physical symptoms or signs objectively caused by stress using tests for endocrine function, immune function, or the autonomic nervous system (ANS). Heart rate variability (HRV) analysis is a reliable, reproducible, and noninvasive method for quantitatively evaluating sympathetic and parasympathetic activity [11]. HRV is known to associate with certain diseases, to relate an emotional condition and a stressful situation [9], and to correlate with mental and psychological states after stress due to various academic examinations in students [12]. However, little research has been done on the HRV-measured stress and its associations with academic achievement of medical students who frequently experience high levels of stress. Previous studies have reported that medical students are exposed to significant stressors during their medical training, and female medical students are particularly more affected than males [10, 1315]. These results suggest that even if the stress is the same, there are various ways of coping with stress due to nonspecific reactions that cause different reactions depending on the subject or situation [3]. Therefore, the authors hypothesized that the stress response in male and female medical students is different and that appropriate stress can increase academic achievement. The purpose of this study was to examine HRV-measured stress in medical students during clinical clerkship and how HRV-measured stress relates to their academic achievement.

\section{Subjects and Methods}

\section{Study Subjects}

A cross-sectional study was conducted. The third year at the $\mathrm{Pu}$ san National University School of Medicine is a clerkship year that includes a required 4-week family medicine rotation. As part of the family medicine clerkship course, all students were asked to measure their own HRV. Then, professors educated students to interpret their HRV results and helped them plan how to manage their stress through interviews and stress management. In this study, we utilized the data of 97 third-year students (participation rate $=77.6 \%$ ) who were nonpregnant and whose HRV was measured between February and November 2016 at the Family Medicine Clinic of Pusan National University Yangsan Hospital. In Korea, medical school begins in February and ends in November. Other data collected included age, gender, anthropometric parameters, smoking status, amount of alcohol consumed, written examination score, and skill examination score. We also conducted individual interviews with students with high stress indices measured by HRV.

\section{Heart Rate Variability}

Data collection and HRV analysis were performed using SA2000E HRV analytic equipment (Medicore Co. Ltd., Seoul, Korea) according to the guidelines of the task force of the European Society of Cardiology and the North American Society of Pacing and Electrophysiology [11]. To control diurnal variation, HRV was measured over a period of $5 \mathrm{~min}$ between 10:00 a.m. and 12:00 p.m. in a quiet environment at room temperature. Participants were asked to abstain from drinking alcohol 1 day before and from smoking or drinking any caffeinated drinks at least $2 \mathrm{~h}$ before HRV testing. Each participant was seated in a chair, and electrodes were placed on the wrists and left foot. The participants were instructed to breathe at a normal rate during HRV measurement. Before the measurement, the participants rested for at least $10 \mathrm{~min}$, breathing spontaneously without talking. The parameters measured in the evaluation of HRV were grouped into 2 categories: time and frequency domains. The mean heart rate and standard deviation of normal-to-normal intervals (SDNNs) were used for time-domain analysis. We also measured the physical stress index (PSI), an indicator that represents the degree of load on the ANS control system. The frequency domain was composed of total spectral power, lowfrequency power (LF, 0.04-0.15 Hz), high-frequency power (HF, $0.15-0.40 \mathrm{~Hz}$ ), and LF/HF ratio. Power in the HF band is an indicator of parasympathetic nervous activity, whereas power in the LF band mostly reflects sympathetic nervous activity. The LF/HF ratio is a general balance indicator used to evaluate sympathetic nervous system/parasympathetic nervous system (SNS/PNS) balance. A high ratio implies intensified activity of SNS or suppression of PNS. LF and HF were represented by 2 graphic views: SNS/PNS and autonomic balance diagrams (ABDs). The stress index was calculated using the HRV parameters proposed in a previous study [16].

Anthropometric Measures and Sociodemographic Factors

BMI was defined as weight $(\mathrm{kg})$ divided by height squared $\left(\mathrm{m}^{2}\right)$. Percentage body fat was assessed by bioelectric impedance analysis (Inbody 720; Biospace Co. Ltd., Seoul, Korea). Participants were classified into underweight $\left(<18.5 \mathrm{~kg} / \mathrm{m}^{2}\right)$, normal weight $(18.5-$ $\left.22.9 \mathrm{~kg} / \mathrm{m}^{2}\right)$, overweight $\left(23-24.9 \mathrm{~kg} / \mathrm{m}^{2}\right)$, or obese $\left(>25 \mathrm{~kg} / \mathrm{m}^{2}\right)$ groups based on BMI according to the Asian-Pacific cutoff points [17]. Smoking status was classified as participants who had never 
Table 1. Basal characteristics of the subjects

\begin{tabular}{lccc}
\hline & Male $(n=48)$ & Female $(n=49)$ & Total $(n=97)$ \\
\hline Smoking status $^{\mathrm{a}}$ & & & \\
$\quad$ Never smoker & $34(70.8)$ & $49(100.0)$ & $55(85.6)$ \\
$\quad$ Ex-smoker & $5(10.4)$ & $0(0.0)$ & $5(5.2)$ \\
$\quad$ Current smoker & $9(18.8)$ & $0(0.0)$ & $9(9.3)$ \\
Excessive drinking $^{\mathrm{b}}$ & $23(47.9)$ & $58.2 \pm 8.2$ & $57.0 \pm 7.5$ \\
Written examination score $_{\text {Skill examination score }}^{\mathrm{c}}$ & $55.7 \pm 6.6$ & $52.7 \pm 10.4$ & $50.4 \pm 9.5$ \\
BMI $^{\mathrm{a}}$ & $48.1 \pm 8.0$ & $20.2 \pm 1.9$ & $22.2 \pm 3.1$ \\
Obesity degree $^{\mathrm{a}}$ & $24.2 \pm 2.7$ & $10(20.4)$ & $10(10.3)$ \\
$\quad$ Underweight $_{\text {Normal }}$ & $0(0.0)$ & $37(75.5)$ & $53(54.6)$ \\
Ovverweight $_{\text {Obese }}$ & $16(33.3)$ & $0(0.0)$ & $18(18.6)$ \\
$\quad$ Body fat percent & $18(37.5)$ & $2(4.1)$ & $16(16.5)$ \\
\hline
\end{tabular}

Data were presented as $n(\%)$ or mean \pm SD. In men and women, excessive alcohol ingestion was defined as $>3$ drinks ( $40 \mathrm{~g})$ and $>2$ drinks ( $20 \mathrm{~g})$ per day, respectively, based on standard drink sizes described in WHO guidelines. ${ }^{\mathrm{a}} p<0.001 .{ }^{\mathrm{b}} p<0.005 .{ }^{\mathrm{c}} p<0.05$ by $\chi^{2}$ test.

smoked (smoked $<100$ cigarettes in their lifetime and not currently smoking), ex-smokers (smoked $\geq 100$ cigarettes in their lifetime and currently a non-smoker), and current smokers (smoked $\geq 100$ cigarettes in their lifetime and currently a smoker). In men and women, excessive alcohol ingestion was defined as $>3$ drinks (40 g) and $>2$ drinks (20 g) per day, respectively, based on standard drink sizes described in WHO guidelines [18]. Academic performance was assessed by summative written and skill examination scores at the end of the third year. The written examination consisted of 400 multiple-choice questions. The score was transformed to a value on a 100-point scale. The skill examination was operated with 12 stations, including 6 clinical performance examination stations and 6 objective structured clinical examination stations. The skill examination score also totals a maximum of 100 points.

\section{Statistical Analysis}

Data are presented as either number (\%) or mean \pm SD. Analysis of continuous variables was performed using 2-sample $t$ test, and categorical variables were analyzed using the $\chi^{2}$ test for comparison of the 2 groups. ANOVA was used to compare means among the 3 groups. Pearson correlation coefficients were used to examine the correlation between summative written or skill examination scores at the end of the third year and other variables. The data were analyzed using IBM SPSS Statistics 24.0 (IBM, Chicago, IL, USA). A p value $<0.05$ was considered statistically significant.

\section{Results}

\section{General Characteristics of Subjects}

The male-to-female ratio was 0.98 . In total, $18.8 \%$ of male students were current smokers, but none of the female students were smokers. The proportion of male stu- dents with heavy drinking was $47.9 \%$, which was 2.6 times higher than that of female students. A significantly higher proportion of male students (29.2\%) than female students (4.1\%) were obese. Most female students were normal or underweight. There was no significant difference in the written examination scores between male and female students at the end of the third year, but the female mean score in skill examinations was more than 4 points higher than the male mean score $(p<0.05$, Table 1$)$.

\section{HRV Parameters}

Female students had an unhealthy tachogram ratio 11.6 times higher than in that in males, unhealthy R-R interval variability 13.6 times higher than that in males, and abnormal ABD 2.7 times higher than that in males. On the other hand, there was no gender difference in the other variables (Table 2).

\section{Stress Variabilities and Academic Achievement}

The correlation coefficient between the written examination score and the skill examination score was 0.370 $(p<0.001)$. Factors correlating positively with the written examination score were PNS $(r=0.218, p=0.033)$, SDNN $(r=0.245, p=0.016)$, SNS/PNS balance $(r=0.218, p=$ $0.033)$, and stress index $(r=0.381, p=0.004)$, while factors that showed significantly negative correlation with the written examinations score were BMI $(r=-0.224, p=$ $0.027)$, obesity degree $(r=-0.194, p=0.016)$, and excessive drinking $(r=-0.245, p=0.016)$. The one factor cor- 
Table 2. Stress-related heart rate variables of study subjects

\begin{tabular}{|c|c|c|c|c|}
\hline & Male $(n=48)$ & Female $(n=49)$ & Total $(N=97)$ & $p$ value \\
\hline \multicolumn{5}{|l|}{ Time domain } \\
\hline SDNN, unhealthy & $10(20.8)$ & $11(22.4)$ & $21(21.6)$ & 0.847 \\
\hline Tachogram, unhealthy & $1(2.1)$ & $12(24.4)$ & $13(13.1)$ & 0.001 \\
\hline Histogram, unhealthy & $28(59.6)$ & $33(67.3)$ & $61(62.9)$ & 0.358 \\
\hline RRV, unhealthy & $1(2.1)$ & $14(28.6)$ & $15(15.5)$ & $<0.001$ \\
\hline PSI, unhealthy & $5(10.4)$ & $7(14.2)$ & $12(12.4)$ & 0.563 \\
\hline \multicolumn{5}{|l|}{ Frequency domain } \\
\hline $\mathrm{TP}$ reduction & $15(31.3)$ & $21(42.9)$ & $36(37.1)$ & 0.237 \\
\hline LF reduction & $11(22.9)$ & $17(34.7)$ & $28(28.9)$ & 0.201 \\
\hline HF reduction & $11(22.9)$ & $18(36.7)$ & $29(29.9)$ & 0.137 \\
\hline \multicolumn{5}{|l|}{$\mathrm{LF} / \mathrm{HF}$ ratio } \\
\hline Low & $5(10.4)$ & $8(16.3)$ & $13(13.4)$ & \\
\hline Normal & $38(79.2)$ & $34(69.4)$ & $72(74.2)$ & 0.539 \\
\hline High & $5(10.4)$ & $7(14.3)$ & $12(12.4)$ & \\
\hline \multicolumn{5}{|l|}{ SNS/PNS balance } \\
\hline SNS dominant & $14(29.2)$ & $17(34.7)$ & $31(32.0)$ & \\
\hline PNS dominant & 7 (14.6) & $11(22.4)$ & $18(18.6)$ & 0.383 \\
\hline Balanced & $27(56.3)$ & $21(42.9)$ & $48(51.5)$ & \\
\hline ABD, abnormal & $4(8.3)$ & $11(22.4)$ & $15(15.5)$ & 0.039 \\
\hline Stress index, high & $9(18.8)$ & $10(20.4)$ & $19(19.6)$ & 0.838 \\
\hline
\end{tabular}

Data were presented as $\mathrm{n}(\%)$. SDNN, standard deviation of the normal-to-normal intervals; RRV, R-R interval variability; PSI, physical stress index; TP, total power; LF, low-frequency group; HF, high-frequency group; SNS, sympathetic nervous system; PNS, parasympathetic nervous system; ABD, autonomic balance diagram.

relating positively with the skill examination score was gender (female; $r=0.240, p=0.018$ ), while BMI ( $r=$ $-0.249, p=0.014$ ) correlated negatively with the skill examination score (Table 3 ).

\section{Academic Achievement by Stress Variabilities}

Students with unhealthy SDNN scored 4 points higher $(p=0.004)$ than those with healthy SDNN in the written examination, but no difference was observed in skill examination scores. In SNS/PNS balance, students with predominantly sympathetic nerve activity had a higher written examination score ( 5 points, $p=0.018$ ) than students with a balance between the sympathetic nerve and parasympathetic nerves $(p=0.018)$; however, no difference was observed in skill examination scores. Students with abnormal ABDs did not differ in the written examination scores compared to students with normal ABDs, whereas students with abnormal ABD scores had a higher skill examination score ( 5 points, $p=0.03$ ) than those with normal ABDs. Students with a higher stress index had a higher written examination score ( 6 points, $p=$ 0.012 ) than students with normal stress, but no difference was seen in the skill examination scores (Table 4).

\section{Discussion}

HRV is a biological marker of stress in humans. Current neurobiological evidence suggests that HRV is impacted by stress and supports its use for the objective assessment of stress [19]. In this study, we aimed to examine the characteristics of HRV in medical students and to ascertain how the stress relates to academic achievement in medical students during clerkship.

Everyone experiences stress; however, even if stress is experienced in similar conditions, the level and duration of stress and physical response of each person to stress are not the same due to individual characteristics [19]. High efficiency of students' intellectual activities can be achieved through high tension mechanisms [3]. Medical students have a high level of stress due to excessive academic load, frequent examination, fear of failure due to pass/fail grading system, peer relations, and financial issues. It is not easy to manage such stresses in real-life situations. Some medical students even suffer from depression, anxiety, psychological distress, or burnout [20]. Although academic pressure is a stress factor, academic achievement is an important indicator of educational 
Table 3. Correlation between stress variabilities and academic achievement

\begin{tabular}{|c|c|c|}
\hline & $\begin{array}{l}\text { Written } \\
\text { examination score }\end{array}$ & $\begin{array}{l}\text { Skill } \\
\text { examination score }\end{array}$ \\
\hline Written examination score & 1 & $0.370(<0.001)$ \\
\hline Skill examination score & $0.370(<\mathbf{0 . 0 0 1})$ & 1 \\
\hline Gender (male 1 , female 2 ) & $0.136(0.183)$ & $0.240(\mathbf{0 . 0 1 8})$ \\
\hline BMI & $-0.224(\mathbf{0 . 0 2 7})$ & $-0.249(\mathbf{0 . 0 1 4})$ \\
\hline Body fat percent & $0.105(0.304)$ & $0.004(0.971)$ \\
\hline Obesity & $-0.194(0.057)$ & $-0.180(0.078)$ \\
\hline Smoking status & $0.036(0.724)$ & $-0.032(0.753)$ \\
\hline Excessive drinking & $-0.245(\mathbf{0 . 0 1 6})$ & $0.007(0.942)$ \\
\hline SDNN & $0.245(\mathbf{0 . 0 1 6})$ & $0.048(0.640)$ \\
\hline Tachogram, unhealthy & $0.045(0.667)$ & $-0.049(0.897)$ \\
\hline Histogram, unhealthy & $0.065(0.532)$ & $0.014(0.897)$ \\
\hline RRV, unhealthy & $0.034(0.740)$ & $-0.024(0.816)$ \\
\hline PSI, abnormal & $0.186(0.071)$ & $-0.006(0.952)$ \\
\hline $\mathrm{TP}$ reduction & $0.034(0.744)$ & $0.072(0.481)$ \\
\hline LF reduction & $-0.034(0.743)$ & $0.076(0.462)$ \\
\hline HF reduction & $0.021(0.836)$ & $0.090(0.378)$ \\
\hline $\mathrm{LF} / \mathrm{HF}$ ratio & $-0.049(0.636)$ & $-0.014(0.891)$ \\
\hline SNS/PNS balance & $0.218(\mathbf{0 . 0 3 3})$ & $0.053(0.608)$ \\
\hline ABD, abnormal & $0.120(0.247)$ & $0.165(0.111)$ \\
\hline Stress index & $0.381(\mathbf{0 . 0 0 4})$ & $0.153(0.266)$ \\
\hline
\end{tabular}

Data were presented as correlation coefficient ( $p$ value). Bold values indicate statistically significant results. SDNN, standard deviation of the normal-to-normal intervals; RRV, R-R interval variability; PSI, physical stress index; TP, total power; LF, low-frequency group; HF, high-frequency group; SNS, sympathetic nervous system; PNS, parasympathetic nervous system; ABD, autonomic balance diagram. Smoking status: 1 never smoked; 2 ex-smoker; 3 current smoker; excessive drinking: 0 no; 1 yes; SDNN: 0 normal, 1 abnormal; tachogram, unhealthy; histogram, unhealthy, RRV, unhealthy; PSI, abnormal; TP reduction; LF reduction; HF reduction; ABD, abnormal: 1 no, 2 yes; LF/HF ratio: 1 low, 2 normal, 3 high; SNS/PNS balance: 1 SNS dominant, 2 PNS dominant, 3 balanced; stress index: 1 normal, 2 high.

outcomes. As reported in previous studies [2, 21], the results of this study showed that stress measured by HRV was also related to academic achievement.

Although appropriate stress is beneficial in stimulating learning and memory in students, previous studies showed mixed results between stress and academic achievement $[6,8,9,22]$. Some studies have reported a negative correlation [6], others have shown a positive correlation [8], and yet others have reported no relationship [9] between stress and academic achievement. A previous study found that optimal stress levels could improve academic achievement, which supports an inverted-U relationship between stress and performance [5]. An analysis of academic achievement according to the stress level
Table 4. Differences of academic achievement by results of stress variabilities

\begin{tabular}{lll}
\hline & $\begin{array}{l}\text { Written } \\
\text { examination } \\
\text { score }\end{array}$ & $\begin{array}{l}\text { Skill } \\
\text { examination } \\
\text { score }\end{array}$ \\
\hline SDNN & & \\
$\quad$ Healthy $(n=76)$ & $55.8 \pm 7.1^{\mathrm{a}}$ & $49.7 \pm 8.8$ \\
$\quad$ Unhealthy $(n=21)$ & $61.2 \pm 8.1^{\mathrm{a}}$ & $52.3 \pm 12.1$ \\
SNS/PNS balance & & \\
$\quad$ SNS dominant $(n=31)$ & $59.5 \pm 7.8^{\mathrm{b}}$ & $50.7 \pm 9.5$ \\
$\quad$ PNS dominant $(n=18)$ & $57.5 \pm 7.8$ & $50.8 \pm 8.6$ \\
$\quad$ Balanced $(n=48)$ & $54.7 \pm 6.2^{\mathrm{b}}$ & $49.6 \pm 9.5$ \\
ABD & $56.6 \pm 7.5$ & $49.8 \pm 8.7^{\mathrm{c}}$ \\
$\quad$ Normal $(n=82)$ & $59.3 \pm 7.5$ & $55.5 \pm 11.4^{\mathrm{c}}$ \\
Abnormal $(n=15)$ & $56.2 \pm 7.2^{\mathrm{d}}$ & $49.6 \pm 8.8$ \\
Stress index & $62.3 \pm 6.0^{\mathrm{d}}$ & $53.0 \pm 11.8$ \\
$\quad$ Normal $(n=78)$ & & \\
High $(n=19)$ & &
\end{tabular}

Data were presented as mean \pm SD. SDNN, standard deviation of the normal-to-normal intervals; SNS, sympathetic nervous system; PNS, parasympathetic nervous system; ABD, autonomic balance diagram. ${ }^{\mathrm{a}} p=0.004 .{ }^{\mathrm{b}} p=0.018 .{ }^{\mathrm{c}} p=0.03 .{ }^{\mathrm{d}} p=0.02$.

among female college students in Pakistan showed that the students with appropriate stress (mildly tense or moderately tense) had higher academic achievement than the very tense (and not tense) students [5]. However, a previous study reported that the precise shape of the invertedU-shape curve may vary for different personal characteristics [22]. As such, there are various results concerning the relationship between stress and academic achievement. The reason why previous research results were inconsistent could partly be explained by the fact that their subjects were different [22] and that researchers relied heavily on self-reported questionnaires as a tool for measuring stress [5-9]. In self-reported questionnaires, it is common to use the average of the factors to be measured, which is somewhat ambiguous for use as concrete guidance in actual educational situations. Therefore, we attempted to analyze and interpret the changes in cognitive and psychological states of learners during the learning process by measuring physiopsychological reaction [23]. Physiopsychological response is an objective indicator, which can explain the state of the object under investigation at various levels without undue interference or influence on learning. Therefore, it is relatively easy to capture the cognitive and psychological states of objects that cannot be observed with the naked eye and allows evaluators to deepen their understanding of learners [23]. It seems 
that when students are not stressed, students do not pay much attention, but as stress increases, they focus on their work and improve their academic performance. However, if stress is sustained, it can have a negative effect on academic achievement, and excessive stress appears to reduce achievement levels [5]. This should be taken into account when teaching or counseling students.

Among the methods for confirming the physiological response, HRV is used as an index to evaluate the physiological adaptability of the subject to a changing environment in various clinical experiments involving changes in the ANS. In addition, the test for HRV is recognized as useful in confirming the ANS activity and balance level in objectively evaluating the psychological and emotional states of an individual [24]. Nonetheless, we were not able to find a study that measured and interpreted HRV in the context of testing or learning. However, the present study using HRV has shown that a certain degree of stress has a positive effect on academic achievement by confirming that SDNN, SNS/PNS balance, and stress index are positively correlated with examination scores. When the stress lasts for a certain length of time, the mind and body can be kept in a balanced state by the principle of homeostasis; thus, stress in the form of a little tension becomes a person's driving force and increases one's efficiency and productivity. This degree of stress can be beneficial both physically and mentally. However, when people are less able to cope with stressors or repeated stress, the ANS activity is reduced and unbalanced, resulting in emotional anxiety and depression, and constant tension of the ANS. As a result, mental or physical dysfunctions or illnesses appear, making it essential to recognize and manage stress for good health. In addition, stress experienced by medical students during their education greatly influences their psychological attitudes as students and as physicians in the future. This can seriously affect the formation of doctor-patient relationships and the quality of medical care [25]. Doctors are exposed to high levels of stressors from emotions that arise in the context of patient care to the situations in which they practice [26]. Nevertheless, the problems for medical students are that stress is a part of the medical schooling that is to be endured in the future, and the level of interest and recognition of the college or professors in the stress of medical students is very low. Therefore, schools need to understand the relationship between HRV-measured stress and academic achievement and provide students with effective counseling and educational programs for stress management based on HRV results.

Medical students, regardless of gender, suffer from many stresses as well as general stress. In general, medical students are constantly undergoing some level of stress. However, in this study, the female students were more stressed. In addition, female students scored better than male students in both written and skilled examinations. Female students are more likely than male students to have higher academic achievement [27], and female students are more likely to perceive stress than male students. Previous studies have shown inconsistent results regarding gender difference in academic performance, but in general, female medical students in Korea and China have significantly higher scores than male students, as supported by the results of the present study $[27,28]$. In previous studies, the gender differences were explained by the findings that female students had higher perceptions of academic performance, internal motivation for academic achievement, and more academic planning activity than male students [13]. In addition, female students were more likely to focus their perceived stress on academic performance than male students [14]. In other words, it may be interpreted that female students tend to react more sensitively than male students to similar situations or to the environment in general. In addition, as female students perceive the amount and the complexity of the workload increases significantly more than the male student, they are more likely to suffer more serious academic stress than male students, thereby experiencing more emotional and physical exhaustion [15].

As described above, this study examined the relationship between stress and academic achievement of medical students using HRV, a biological marker of stress. As a result, it was found that the higher the stress level, the higher the students' paper-written test scores, and that there was a gender difference in the stress level. These findings reveal that stress is not a factor that negatively affects all medical school students collectively but that stress can also act partially positively on students. Stress may have a positive effect, at least partially, on the academic achievement of students. In addition, this study has suggested that understanding students' stress levels can help them manage school and academic achievement. In particular, this study is meaningful in that it attempted more objective and scientific measurements of psychological construct by measuring stress using biological markers, rather than self-administered questionnaires in the context of clinical clerkship.

Even at the same level of stress, ways of coping with stress can be different. In a previous study in Korea, the most common coping styles of medical students were the avoidance and problem-focused coping strategy [29]. These 2 are known as conflicting strategies. Students who better adapt to stressful situations used problem-focused 
coping strategies, and students with low level of adaptation and high depression used avoidance coping strategies [29]. Stress coping styles can be diverse and culturally different. Therefore, it will be necessary to guide and support each student to have a healthy coping method considering cultural and individual characteristics so that they can achieve academic achievement and adapt well.

Our study has several limitations. First, each medical school has its own characteristics and rules which decide teaching and learning methods. These differences may limit the generalizability of our findings, even though a high proportion of third-year students participated in this study. Second, HRV was measured only once in this study. The degree of stress may vary depending on when the HRV assessment is performed because students need a period of adaptation to settle into the pace and expectations of the clerkship year. Also, stress as measured by HRV does not only mean stress associated with schoolwork but also reflects everyday life and general health. Nevertheless, HRV represents the overall autonomic context, and previous studies revealed that at rest, HRV indicators have good reliability, repeatability, and reproducibility $[11,17]$. Especially, good reproducibility was shown when HRV was measured repeatedly over 12 -month intervals in patients with type 1 diabetes mellitus [30]. Therefore, HRV at rest can be considered as the usual stress, and previous studies have measured HRV once as in this study. Third, this study aimed to increase the validity of academic stress measurement by measuring the stress level of students in the same clinical clerkship. However, in order to investigate the level of stress of students and how they respond to various clinical clerkship situations, such as on rounds and in the operating room, patient management and in-depth qualitative research through tools such as observation and interview should be conducted in the future. Fourth, the cross-sectional nature of the study prevented us from confirming the impacts of stress-measured HRV in academic performance. In the present study, we are unable to infer causality from our findings. There is still controversy regarding stress and learning. During learning, stress can improve memory formation (leading to stronger memories), while also compromising your memory (leading to the risk of dropping out of the test [21]. Fourth, stress is a very complicated concept. It would be difficult to determine the presence of stress and its severity by measuring HRV alone. Finally, this study was conducted at a single site. Despite these limitations, the strengths of this study were that students' stress was measured more objectively using HRV, instead of a subjective questionnaire; also, academ- ic performance was a comprehensive evaluation (the written examination and the skill examination), not a one-time evaluation.

\section{Conclusion}

Medical students with higher stress measured by HRV were found to have higher academic achievement, especially in written examinations. Female students are more likely to suffer academic stress than male students. This suggests that appropriate stress can have a positive impact on school performance in medical students. However, replication of this study will be needed in order to confirm the results of this first-stage study to measure medical students' stress using HRV, and additional studies are required to assess the long-term effects of HRV-measured stress on medical education.

\section{Statement of Ethics}

As this study used pre-existing, de-identified data, it was exempt from the institutional review board (IRB No. 05-2017-102).

\section{Conflict of Interest Statement}

There are no conflicts of interest.

\section{Funding Sources}

The authors did not receive any funding.

\section{Author Contributions}

Yoo H.H. and Lee S.Y. conceived and designed the study, collected the data, and wrote the paper. Yune S.J., Kam B.S., and Im S.J. collected the data and analyzed the data. All authors discussed the results and contributed to the final manuscript.
References
1 Ishak W, Nikravesh R, Lederer S, Perry R, Ogunyemi D, Bernstein C. Burnout in medi- cal students: a systematic review. Clin Teach. 2013;10(4):242-5.
2 Sohail N. Stress and academic performance among medical students. J Coll Physicians Surg Pak. 2013;23(1):67-71.
3 Stepanova GK, Ustinova MV. Psychological and vegetative characteristics of students: ya- kuts with different educational success. Wiad Lek. 2015;68(4):469-75.
4 Chipidza FE, Wallwork RS, Stern TA. Impact of the doctor-patient relationship. Prim Care Companion CNS Disord. 2015;17(5). 
5 Inam QU, Shireen E, Haider S, Haleem DJ. Perception of academic examination stress: effects on serum leptin, cortisol, appetite and performance. J Ayub Med Coll Abbottabad. 2011;23(2):97-9.

6 Crego A, Carrillo-Diaz M, Armfield JM, Romero M. Stress and academic performance in dental students: the role of coping strategies and examination-related self-efficacy. J Dent Educ. 2016;80(2):165-72.

7 Shim EJ, Jeon HJ, Kim H, Lee KM, Jung D, Noh HL, et al. Measuring stress in medical education: validation of the Korean version of the higher education stress inventory with medical students. BMC Med Educ. 2016; 16(1):302.

8 Kötter T, Wagner J, Brüheim L, Voltmer E. Perceived Medical School stress of undergraduate medical students predicts academic performance: an observational study. BMC Med Educ. 2017;17:256.

9 Dendle C, Baulch J, Pellicano R, Hay M, Lichtwark I, Ayoub S, et al. Medical student psychological distress and academic performance. Med Teach. 2018;40(12):1257-63.

10 Saxena Y, Shrivastava A, Singhi P. Gender correlation of stress levels and sources of stress among first year students in a medical college. Indian J Physiol Pharmacol. 2014; 58(2):147-51.

11 Cipryan L, Litschmannova M. Intra-day and inter-day reliability of heart rate variability measurement. J Sports Sci. 2013;31(2):150-8.

12 Park JE, Lee JY, Kang SH, Choi JH, Kim TY, So HS, et al. Heart rate variability of chronic posttraumatic stress disorder in the Korean veterans. Psychiatry Res. 2017;255:72-7.
13 Nguyen HV, Giang TT. Gender difference in academic planning activity among medical students. PLoS One. 2013;8(2):e55845.

14 Alzahrani SS, Soo Park Y, Tekian A. Study habits and academic achievement among medical students: a comparison between male and female subjects. Med Teach. 2018; 40(Suppl 1):S1-9.

15 Burger PHM, Scholz M. Gender as an underestimated factor in mental health of medical students. Ann Anat. 2018;218:1-6.

16 Joeng HC, Choi YJ. The effect of cold-water immersion on fatigue, stress, and autonomic nervous system activity of body fatigue recipient. Adv Sci Technol Lett. 2015;116:6-10.

17 Pan WH, Yeh WT. How to define obesity? Evidence-based multiple action points for public awareness, screening, and treatment: an extension of Asian-Pacific recommendations. Asia Pac J Clin Nutr. 2008;17(3):370-4.

18 Pearson MR, Kirouac M, Witkiewitz K. Questioning the validity of the $4+/ 5+$ binge or heavy drinking criterion in college and clinical populations. Addiction. 2016;111(10): 1720-6.

19 Kim HG, Cheon EJ, Bai DS, Lee YH, Koo BH. Stress and heart rate variability: a meta-analysis and review of the literature. Psychiatry Investig. 2018;15(3):235-45.

20 Rotenstein LS, Ramos MA, Torre M, Segal JB, Peluso MJ, Guille C, et al. Prevalence of depression, depressive symptoms, and suicidal ideation among medical students: a systematic review and meta-analysis. JAMA. 2016; 316(21):2214-36
21 Vogel S, Schwabe L. Learning and memory under stress: implications for the classroom. NPJ Sci Learn. 2016;1:16011.

22 Salehi B, Cordero MI, Sandi C. Learning under stress: the inverted-U-shape function revisited. Learn Mem. 2010;17(10):522-30.

23 Dirican AC, Göktürk M. Psychophysiological measures of human cognitive states applied in human computer interaction. Procedia Comput Sci. 2011;3:1361-7.

24 Bali A, Jaggi AS. Clinical experimental stress studies: methods and assessment. Rev Neurosci. 2015;26(5):555-79.

25 Shapiro SL, Shapiro DE, Schwartz GE. Stress management in medical education: a review of the literature. Acad Med. 2000;75(7):74859.

26 Yates SW. Physician stress and burnout. Am J Med. 2020;133(2):160-4.

27 Ryue S, Lee H, Jeon WT. The relationship among the learning motivation, the characteristics of multiple intelligence and academic achievement in medical school students. Korean Med Educ Rev. 2013;15(1):46-53.

28 Zhou YX, Zhao ZT, Li L, Wan CS, Peng CH, Yang J, et al. Predictors of first-year GPA of medical students: a longitudinal study of 1285 matriculates in China. BMC Med Educ. 2014; 14:87.

29 Ko J, Yoon T-Y, Park J. The effects of coping style on subjective well-being among medical students. Korean J Med Educ. 2007;19(3): 225-33.

30 Burger AJ, Charlamb M, Weinrauch LA, D'Elia JA. Short- and long-term reproducibility of heart rate variability in patients with long-standing type I diabetes mellitus. Am J Cardiol. 1997;80(9):1198-202. 